\title{
Amplitude equations for Rayleigh-Bénard convective rolls far from threshold
}

\author{
P. C. Dauby* and Th. Desaive \\ Université de Liège, Institut de Physique B5, B-4000 Liège 1, Belgium \\ J. Bragard \\ Department of Physics and Center for Interdisciplinary Research on Complex Systems, Northeastern University, \\ Boston, Massachusetts 02115 \\ P. Cerisier \\ IUSTI, UMR CNRS 6595, Université de Provence, Technopôle Château-Gombert, Rue E. Fermi 5, F-13453 Marseille Cedex 13, France
}

(Received 13 February 2001; published 9 November 2001)

\begin{abstract}
An extension of the amplitude method is proposed. An iterative algorithm is developed to build an amplitude equation model that is shown to provide precise quantitative results even far from the linear instability threshold. The method is applied to the study of stationary Rayleigh-Bénard thermoconvective rolls in the nonlinear regime. In particular, the generation of second and third spatial harmonics is analyzed. Comparison with experimental results and direct numerical calculations is also made and a very good agreement is found.

DOI: 10.1103/PhysRevE.64.066301

PACS number(s): 47.20.Bp, 05.45.-a, 44.25.+f, 47.27.Te
\end{abstract}

\section{INTRODUCTION}

Rayleigh-Bénard (RB) convection [1,2] is an illuminating example of pattern formation outside of equilibrium. Numerous studies have been devoted to this system. Some of them are concerned with the nonlinear competition between different structures that develop above the linear convective threshold. For instance, the stability domains of rolls, hexagons, or square cells have been determined in terms of the different parameters of the system [3-5]. The transition to more complex, nonstationary, behaviors has also been analyzed [6]. Interesting general references to the RB problem may be found, for instance, in the books by Koschmieder [1] and by Colinet et al. [2].

Navier-Stokes equations, and, more generally, the equations of continuum mechanics are nonlinear. More precisely, in the simplest cases, the nonlinearities are quadratic and originate in the advective terms of the material time derivatives. For this reason, when a spatial structure appears in the solution of these equations, its second-order harmonics are directly excited through the quadratic terms and may influence the observed pattern. An interesting illustration of the influence of the second-order harmonics has been recently presented by Regnier et al. [5] who examined the interface deformations of an hexagonal pattern in coupled gravitydriven and capillary thermoconvection. They showed that when the depth of the fluid layer is such that the deformations of the upper free surface due to gravity and due to capillarity exactly compensate for the linearly unstable mode, the interface relief in the nonlinear regime is determined by the second-order harmonics only ("hybrid" relief).

One motivation of the present work is the experimental evidence of the appearance of not only second-, but also third-order harmonics in a thermoconvective roll pattern in the RB problem. This problem has already been approached

\footnotetext{
*Electronic address: PC.Dauby@ulg.ac.be
}

by several authors in the past (see, for instance, [7] and references therein). In [8], experimental results are reported and a perturbation analysis limited to third-order terms and based on the Malkus-Veronis approach $[9,10]$ is presented and compared with experiments. Pure numerical calculations [11] and a double Fourier expansion in $x$ and $z$ combined with a Galerkin method [7] are also shown to display the appearance of the second- and third-order harmonics of the basic sine roll pattern.

In this paper, we propose an extension of the amplitude method used in many nonlinear studies on thermoconvection (see [2] and references therein). From a theoretical point of view, the original amplitude method is valid only asymptotically close to the linear stability limit. Unfortunately, this method has sometimes been used to analyze nonlinear regimes that are not close to the threshold. For instance, the depth of the subcritical convection domain in Marangoni convection and the transitions between rolls, hexagonal cells, and square patterns were determined in this context [3-5]. The value of the control parameter corresponding to these bifurcations is not always small, which makes these results questionable, from a quantitative, but also from a qualitative, point a view.

In this paper, we develop an extended amplitude formalism to allow a rigorous analysis of convection further from the linear threshold. For simplicity and clarity, the formalism, which is based on an iterative algorithm, is presented here through a detailed analysis of Rayleigh-Bénard thermoconvective rolls in the nonlinear regime. More precisely, we show that the extended method permits us to account in an easy way for the experimental observations of the second and third spatial harmonics. The validity of the method far from the threshold is checked by confronting it to experimental data and also direct numerical simulations.

Our experimental set-up is described in details in [12]. Let us summarize the description of the apparatus (Fig. 1). A finite rectangular container with dimensions $12 \times 3 \times 1 \mathrm{~cm}^{3}$ is filled up with a Rhodorsil 47V100 silicone oil and heated 


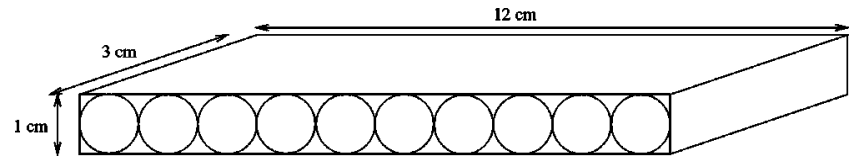

FIG. 1. Sketch of the experimental setup

from below. The upper and lower boundary of the fluid are two horizontal glass plates with a thickness of $0.3 \mathrm{~cm}$. By measuring the deflection of a laser beam shining through the fluid, the overall horizontal temperature gradient is determined [12]. The velocity field is also measured using PIV. For a relative distance to the linear convective threshold slightly larger than 1 , second- and third-order harmonics clearly appear in the horizontal temperature gradient and velocity field.

In Sec. II, the basic governing equations for the RB problem are recalled and the classic nonlinear amplitude method is briefly described. Section III contains a qualitative explanation of the presence of secondary harmonics based on an amplitude model with only four modes. In Sec. IV, we present the extended amplitude method, which is the main result of the paper. It consists in a precise strategy to build an amplitude model valid even far from threshold. We compare the solution given by this model with direct numerical calculations. We show that in the specific case of RB convection, a model with 11 amplitude equations gives very good quantitative agreement with numerics. We present our experimental results in Sec. V and again compare these results with the solution of our model. Conclusions are drawn in the last section.

\section{BASIC EQUATIONS AND BASIS OF THE NONLINEAR METHOD}

Let us consider, for simplicity, a horizontally infinite fluid layer that is contained between two rigid horizontal boundaries. The equations for this system are well known $[1,2]$. When the Boussinesq hypotheses hold, the perturbations with respect to the conductive solution obey the following set of partial differential equations:

$$
\begin{gathered}
\boldsymbol{\nabla} \cdot \mathbf{u}=0, \\
\operatorname{Pr}^{-1}\left[\partial_{t} \mathbf{u}+(\mathbf{u} \cdot \boldsymbol{\nabla}) \mathbf{u}\right]=-\nabla p+\nabla^{2} \mathbf{u}+\operatorname{Ra} T \mathbf{e}_{z}, \\
\partial_{t} T+\mathbf{u} \cdot \boldsymbol{\nabla} T-w=\nabla^{2} T .
\end{gathered}
$$

We have chosen the vertical $z$ axis in the direction opposite to gravity. The equations are written in a dimensionless form with lengths scaled by the thickness $d$ of the liquid layer. The time scale is $d^{2} / \kappa$, with $\kappa$ the heat diffusivity of the liquid. The velocity is scaled by $\kappa / d$ and the temperature scale is chosen as $\beta d$, where $\beta$ is the vertical temperature gradient that would exist in a purely conductive state. Symbols $\mathbf{u}$ $=(u, v, w), p$, and $T$ represent the dimensionless perturbations (with respect to the conductive solution) of the velocity, pressure, and temperature fields. The Rayleigh and Prandtl numbers $\mathrm{Ra}$ and $\mathrm{Pr}$ are defined as

$$
\begin{gathered}
\mathrm{Ra}=\frac{g \alpha \beta d^{4}}{\nu \kappa}, \\
\operatorname{Pr}=\frac{\nu}{\kappa},
\end{gathered}
$$

where $\nu$ and $\alpha$ are the liquid kinematic viscosity and the coefficient of thermal expansion, respectively.

The boundary conditions for the velocity perturbations at the bottom $(z=0)$ and at the top $(z=1)$ of the layer express the no-slip condition along the horizontal plates. For the temperature perturbations, a general Biot condition describes the heat exchanges through the boundaries. The corresponding equations are

$$
\begin{gathered}
\mathbf{u}=0 \text { at } z=0 \text { and at } z=1, \\
-\frac{\partial T}{\partial z}+\mathrm{Bi}_{0} T=0 \text { at } z=0, \\
\frac{\partial T}{\partial z}+\mathrm{Bi}_{1} T=0 \text { at } z=1,
\end{gathered}
$$

where $\mathrm{Bi}_{0}$ and $\mathrm{Bi}_{1}$ denote the Biot numbers at the bottom and the top of the layer, respectively. In the experiments that we are considering in the present paper, these Biot numbers are equal since the glass plates at $z=0$ and $z=1$ are the same. The value $\mathrm{Bi}=46$ is determined by using the expression [13] $\mathrm{Bi}=\left(\lambda_{\text {wall }} / \lambda\right)\left[k_{c} / \tanh \left(k_{c} d_{\text {wall }} / d\right)\right]$ where $\lambda_{\text {wall }}$ $=1.74 \mathrm{Wm}^{-1} \mathrm{~K}^{-1}$ and $d_{\text {wall }}=0.3 \mathrm{~cm}$ are the heat conductivity and thickness of the glass plates, while $\lambda$ $=0.16 \mathrm{Wm}^{-1} \mathrm{~K}^{-1}$ is the heat conductivity of the silicone oil. The corresponding dimensionless critical wave number and Rayleigh numbers are given by $k_{c}=3.07$ and $R a_{c}$ $=1653$, respectively (a standard spectral-tau method $[14,15]$ was used for the calculations of the critical parameters).

The starting point of our nonlinear method is the amplitude method that has been described in details, for instance, in $[3,2]$. Two-dimensional rolls are the only convective pattern observed in the present experiments, therefore, the general three-dimensional method is simplified here into its twodimensional (2D) counterpart. Let us recall the main steps of the method. First, the Rayleigh number is fixed to its critical value $\mathrm{Ra}_{c}$ and the eigenmodes of the linearized equations are numerically determined by a spectral tau method, with the growth rate $\sigma_{p}$ of the perturbations as eigenvalue parameter. Using complex notations, the 2D eigenfunctions are

$$
\begin{aligned}
& \mathbf{u}_{k, p}=\mathbf{U}_{p}(z) \exp (i k x) \exp \left(\sigma_{p} t\right), \\
& T_{k, p}=\theta_{p}(z) \exp (i k x) \exp \left(\sigma_{p} t\right),
\end{aligned}
$$

where $k$ is the horizontal wave number and $x$ the horizontal coordinate. It is important to note that for each value of $k$, an infinite set of eigenvalues exists for the growth rate, with corresponding vertical eigenfunctions $\mathbf{U}_{p}(z)$ and $\theta_{p}(z)$ for the velocity and temperature perturbations. The maximum value of the growth rate is zero and corresponds to a wave number equal to the critical value $k_{c}$. The index $p$ runs from 
one to infinity and numbers the negative growth rates, which are assumed to be ordered in such a way that the real part of $\sigma_{p}$ decreases with $p$ (for $k=k_{c}$, one has $\sigma_{1}=0$ ). The eigenmodes are normalized in such a way that the maximum value of the modulus of $\theta_{p}(z)$ is equal to 1 .

The solution of the nonlinear equations is then expressed as the following series depending on the eigenmodes $\left(\mathbf{u}_{P}, T_{P}\right)$ :

$$
\left(\begin{array}{c}
\mathbf{u} \\
T
\end{array}\right)=\sum A_{P}(t)\left(\begin{array}{c}
\mathbf{u}_{P} \\
\frac{R a_{c}}{R a} T_{P}
\end{array}\right)+\text { c.c. }
$$

where $P$ is written for $k, p$, and the $A_{P}(t)$ are the complex time-dependent amplitudes. It is worth emphasizing that the spatial functions used in this decomposition are not exactly the eigenmodes of the physical problem, due to the rescaling factor $\mathrm{Ra}_{c} / \mathrm{Ra}$. However, for each value of $\mathrm{Ra}$, these functions $\mathbf{u}_{P}$ and $\left(\mathrm{Ra}_{c} / \mathrm{Ra}\right) T_{P}$ may be considered as the eigenmodes of a "mathematical" eigenvalue problem, which is easily obtained from the original problem by letting this factor explicitly appear. These spatial modes may thus be considered as independent. It may also be checked that the adjoint eigenmodes of this problem are given by $\left(\mathbf{u}_{P}^{\star},\left(\mathrm{Ra} / \mathrm{Ra}_{c}\right) T_{P}^{\star}\right)$, where the $\left(\mathbf{u}_{P}^{\star}, T_{P}^{\star}\right)$ are the adjoint eigenmodes of the original problem. From a more physical point of view, the introduction of the rescaling factor $\mathrm{Ra}_{c} / \mathrm{Ra}$ in Eq. (11) may be justified by noting that the temperature scale used in Eqs. (1)-(3) is $\beta d$ while the "critical scale" $\beta_{c} d$ is used to determine the eigenmodes (10). When the unknown fields are written under the form (11), the incompressibility Eq. (1) and the boundary conditions (6)-(8) are automatically satisfied. The series (11) is then introduced in Eqs. (2)-(3), which are then projected on the adjoint eigenfunctions of the "mathematical" problem introduced above. The momentum equation is thus multiplied by $\mathbf{u}_{P}^{\star}$, the velocity field of the adjoint eigenvalue problem and the energy equation is multiplied by $\left(\mathrm{Ra} / \mathrm{Ra}_{c}\right) T_{P}^{\star}$, with $T_{P}^{\star}$ the temperature field of the adjoint eigenvalue problem. Both relations are added and integrated over the fluid volume. When the biorthogonality relations between the solutions of the eigenvalue problem and its adjoint are used, together with the incompressibility condition and the boundary conditions, we get the following evolution equations for the amplitudes:

$$
\frac{d A_{P}}{d t}=\sigma_{P} A_{P}+\epsilon \sum_{Q} M_{P Q} A_{Q}+\sum_{Q, L} N_{P Q L} A_{Q} A_{L} .
$$

In this equation, $\epsilon=\left(\mathrm{Ra}-\mathrm{Ra}_{c}\right) / \mathrm{Ra}_{c}$ is the relative distance to the threshold and the matrices $M$ and $N$ are given by

$$
\begin{gathered}
M_{P Q}=\frac{\left\langle\theta_{P}^{\star} w_{Q}\right\rangle}{\left\langle\theta_{P}^{\star} \theta_{P}+\operatorname{Pr}^{-1} \mathbf{u}_{P}^{\star} \cdot \mathbf{u}_{P}\right\rangle}, \\
N_{P Q L}=-\frac{\left\langle\theta_{P}^{\star}\left(\mathbf{u}_{Q} \cdot \nabla \theta_{L}\right)+\operatorname{Pr}^{-1} \mathbf{u}_{P}^{\star} \cdot\left[\left(\mathbf{u}_{Q} \cdot \nabla\right) \mathbf{u}_{L}\right]\right\rangle}{\left\langle\theta_{P}^{\star} \theta_{P}+\operatorname{Pr}^{-1} \mathbf{u}_{P}^{\star} \cdot \mathbf{u}_{P}\right\rangle},
\end{gathered}
$$

where the angular bracket is used to denote integration over the fluid volume.

Using a slaving principle $[16,17]$, the infinite dimensional set of Eqs. (12) may be reduced to a finite number of ordinary differential equations for the amplitudes of the most unstable modes of convection. The procedure leading to this reduced system may be briefly summarized as follows. First, the infinite number of eigenmodes is split into two categories. The "basic" modes are the most unstable modes, with the real part of the growth rates close to zero while the "slaved" or "stable" modes are quite damped, due to quite negative values for $\operatorname{Re}\left(\sigma_{P}\right)$. These slaved modes are present in the solution only as the quadratic response to the nonlinear growth of the basic modes above the threshold and their own dynamics may be neglected. For this reason, the time derivative is set to zero in the evolution Eqs. (12) for their amplitudes. This results in an algebraic relation between the basic and slaved amplitudes. When the amplitudes of the slaved modes are small with respect to the basic ones, the quadratic terms of these algebraic equations containing only damped modes may be neglected and the following expression of the slaved amplitudes in terms of the basic ones may easily be deduced:

$$
A_{P_{s}}=-\frac{1}{\sigma_{P_{s}}} \sum_{Q, L} N_{P_{s} Q L} A_{Q} A_{L},
$$

where the subindex $s$ indicates that a slaved mode is considered. In the right-hand side (r.h.s.) of Eq. (15), the indices $Q$ and $L$ refer to basic modes only. Note that this relation, based on the smallness of $A_{P_{s}}$, is always correct close to the threshold where the amplitudes of the basic modes can be assumed sufficiently small (at least when the bifurcation is supercritical, which is actually the case for rolls).

Expressions (15) for the amplitudes of the slaved modes may then be introduced in the evolution Eqs. (12) for the basic modes. If terms of order higher than three are neglected, the following final "amplitude equations" for the amplitudes of the basic modes are obtained:

$$
\begin{aligned}
\frac{d A_{P}}{d t}= & \sigma_{P} A_{P}+\epsilon \sum_{Q} M_{P Q} A_{Q}+\sum_{Q, L} N_{P Q L} A_{Q} A_{L} \\
& +\sum_{Q, L, R} T_{P Q L R} A_{Q} A_{L} A_{R} .
\end{aligned}
$$

In this relation, all amplitudes and indices correspond to basic modes only and the definition of the matrix $T$ is easily deduced from the context. Note also that in practice only a finite number of slaved modes is considered to deduce Eq. (16). Equations (15) show that the amplitudes of the slaved modes decrease with $p$ since the modulus of the growth rate increases with $p$. In the RB problem, the number $N_{s l}(k)$ of slaved modes that are taken into account for each value of $k$ is limited to four or five, which is sufficient to ensure convergence of the coefficients of the amplitude equations, as shown in [3] and checked again in this paper.

To finish this section, let us recall that the Eqs. (16) should be considered as a valid model for the nonlinear con- 




FIG. 2. Stream function (thick lines) and temperature field (thin lines) in a plane perpendicular to the rolls for $\epsilon=2.91$.

vection only if the slaved amplitudes remain small. Otherwise, terms of order higher than three should be taken into account.

\section{SECOND- AND THIRD-ORDER HARMONICS IN THE NONLINEAR REGIME}

The simplest model of nonlinear roll convection consists in a unique amplitude equation for the linearly unstable mode. The quadratically generated slaved modes are then all modes $(p \geqslant 1)$ with $k=0$ and $k=2 \times k_{c}$. Since the modes with $k=3 \times k_{c}$ are absent from the description, this model is unable to provide any account of the presence of the third harmonics observed in experiments and obviously has to be extended.

To describe this experimental evidence, we must increase (first in a heuristic way) the number of modes entering the amplitude Eq. (16). This amounts not to use the simplified representation (15) for some of the slaved and quadradically generated modes but rather include them in the basic modes. Here as basic modes we take not only the critical one but also the three modes with $k=0,2$, and $3 \times k_{c}$, and $p=1$. The first two additional modes are the direct quadratic response to the nonlinear growth of the critical mode while the mode with $k=3 \times k_{c}$ is present because of the quadratic interactions of the two modes $k=1$ and $2 \times k_{c}$. This choice of the basic modes is dictated by the experimental observations and may be considered as somewhat arbitrary from a theoretical point of view. However, in the next section, we develop a precise strategy to select the necessary basic modes.

The modes that are generated by the quadratic interactions of these four basic modes, which we call secondary modes, consist of the eigenmodes with $k=0,1,2$, or $3 \times k_{c}$ and $p$ $>1$ as well as all the modes $(p \geqslant 1)$ with $k=4,5$, and 6 $\times k_{c}$. The real part of the growth rate for these secondary modes is of course negative and their dynamics is neglected. If a simplified expression (15) is used to express the secondary modes, four amplitude equations (16) may be determined for $A_{0}=A_{0 \times k_{c}, 1}, \quad A_{1}=A_{k_{c}, 1}, \quad A_{2}=A_{2 \times k_{c}, 1}, \quad$ and $A_{3}$ $=A_{3 \times k_{c}, 1}$.

The stationary solution of these equations is then used to reconstruct the unknown fields and represent the velocity and

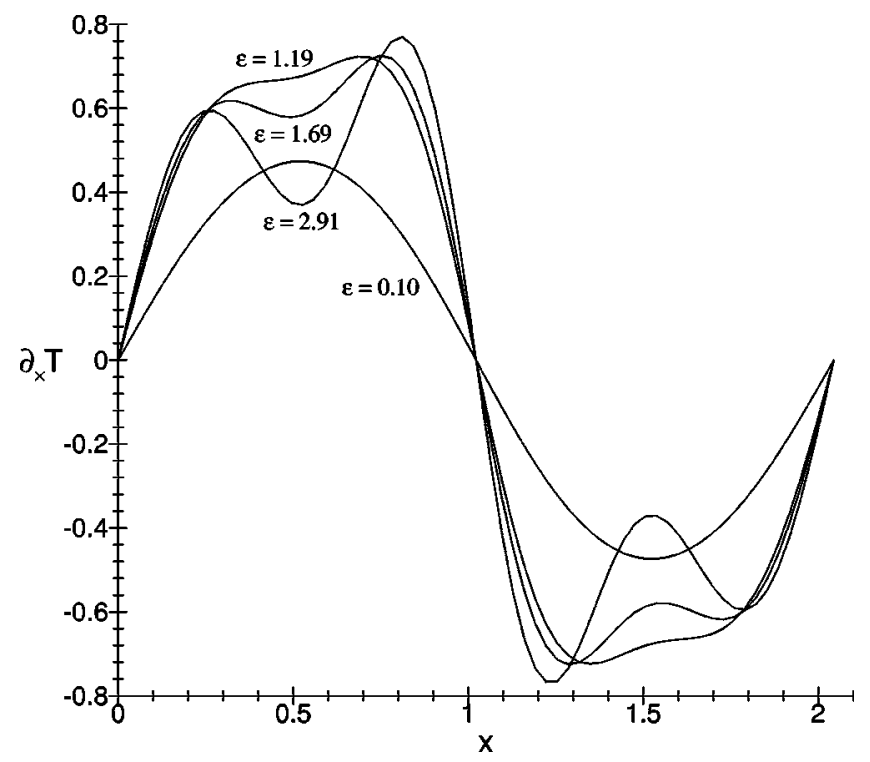

FIG. 3. Dimensionless horizontal temperature gradient $\partial_{x} T$ versus $x$ at $z=0.45$ and for $\epsilon=0.1,1.19,1.69$, and 2.91.

temperature in the fluid layer as shown in Fig. 2. The deformation of the rolls due to the presence of secondary harmonics is clearly seen in the picture. In order to interpret the results of our measurements for the horizontal temperature gradients, we also plot in Fig. 3 the reconstructed horizontal temperature gradient $\partial_{x} T$ at $z=0.45$ for $\epsilon=0.1,1.19,1.69$, and 2.91 (again obtained using the four amplitudes model). Close to the threshold, the curve is almost a sine function while it becomes more and more deformed as $\epsilon$ is further increased. The deformations are due to the increasing importance of the second and third harmonics of the mode $k=k_{c}$. In particular, for the horizontal temperature gradient, the third harmonics appears clearly for $\epsilon$ close to 1.19 . It is also interesting to note that due to the symmetry of the physical system with respect to $z=0.5$ the second harmonics disappears at midheight, as one can see in Fig. 4 where the local extrema due to the third harmonics have all the same abscissa.

\section{GENERALIZED AMPLITUDES METHOD AND COMPARISON WITH NUMERICAL CALCULATIONS}

The results given in the previous section are in good qualitative agreement with experiments and numerical simulations. However, it is possible to improve further, i.e., also get a quantitative agreement. In this section, we build a precise strategy to select the relevant modes that enter the amplitude equations.

The method developed in Sec. II and leading to Eq. (16) is valid only when the quadratically generated secondary modes have small amplitudes. When $\epsilon$ is increased, this condition is not satisfied and the model is no longer accurate.

Let us first consider a unique amplitude equation for the linearly unstable mode. For the largest value of $\epsilon$ that we have to deal with in the problem (this value is around three in our case), we solve the amplitude equation and determine the value of the amplitude of the basic mode. Then, we cal- 


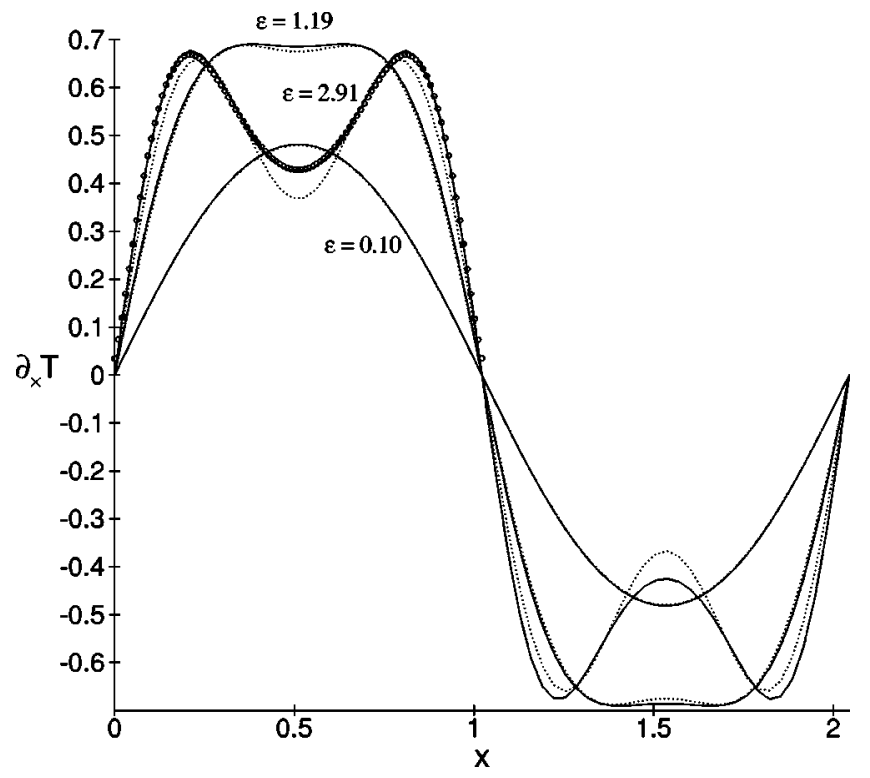

FIG. 4. Dimensionless horizontal temperature gradient $\partial_{x} T$ versus $x$ at $z=0.5$ and for $\epsilon=0.1,1.19$, and 2.91. The dotted and solid lines have been calculated by using 4 and 11 amplitude equations, respectively. The dots correspond to direct numerical calculations (AQUILON code).

culate the values of the secondary amplitudes using Eq. (15) and check the consistency of the results with the fact that the amplitudes of the secondary modes must remain small with respect to the amplitudes of the basic ones. If a secondary amplitude is larger than some small fraction $\left(10^{-2}\right.$ in our calculations) of the largest amplitude of the basic modes, then we have to include this mode in the basic modes in the next model. This procedure is in fact equivalent to considering terms of order higher than three in Eq. (16) but is much easier. After determining the basic modes of the model, the secondary modes are determined as before: their horizontal wave numbers are equal to the sum or the difference of any two wave numbers of the basic modes. Moreover, for each value of $k$, it is sufficient to fix $N_{s l}(k)=4$ or 5 to have convergence of the cubic coefficients of the amplitude equations. This iterative improvement of the model is repeated until all secondary modes have small amplitudes. In the RB problem studied in this paper, this iterative scheme results in a "generalized" 11 amplitude equation model, with, respectively, $3,3,2,2$, and 1 equations for the amplitudes of the most unstable modes with $k=0,1,2,3$, and $4 \times k_{c}$.

The comparison of our theoretical approach and numerical calculations is presented in Fig. 4 where the horizontal temperature gradient is given as a function of $x$ for $z=0.5$ and for different values of $\epsilon(\epsilon=0.1,1.19$, and 2.91). The solid curves correspond to the 11 amplitude equation model while the results for four equations are represented with dotted lines. When $\epsilon$ is not too large, the two curves coincide almost perfectly as expected. Far from threshold, the difference becomes significant. We have performed a direct numerical simulation of Eqs. (1)-(3) using the AQUILON code (finite volumes PDE solver developed at the MASTERENSCPB, Bordeaux, France) for $\varepsilon=2.91$ and the results are represented by the dots in Fig. 4. The agreement between the direct numerical simulations and the 11 amplitude equation model is excellent and provides a confirmation of the validity of the generalized amplitude equations far from threshold. The iterative procedure is very appealing because it gives a much better understanding of which are the physical relevant convective modes.

\section{COMPARISON WITH EXPERIMENTS}

We have carried out different experiments to validate the extended amplitude equation model. For each given experimental situation, the value of $\epsilon$ has been determined in terms of the thermophysical parameters and the applied temperature difference between the top and bottom glass plates. The temperature difference across the fluid layer is measured by thermocouples located at the top and bottom surfaces of the fluid. Since the estimated Biot number is very large (Bi $=46$ ), the top and bottom plates are almost perfect heat conductors and the conductive temperature difference $\beta d$ is almost equal to the experimental temperature difference. Therefore, the experimental Rayleigh number (4) is evaluated directly in terms of the temperature measurement. The coefficient of thermal expansion of the Rhodorsil $47 \mathrm{~V} 100$ silicone oil used in experiments is $\alpha=9.54 \times 10^{-4}$ $\mathrm{K}^{-1}$. The thermal conductivity was given above $(\lambda=0.16$ $\mathrm{Wm}^{-1} \mathrm{~K}^{-1}$ ) and the specific heat at constant pressure is $c=1454.4 \mathrm{~J} \mathrm{~K}^{-1} \mathrm{~kg}^{-1}$. Since the experiments are carried out in different temperature ranges, the variations of the density and viscosity with $T$ are taken into account, and we take the value corresponding to the mean temperature of the experiment. The phenomenological laws for these variations are given by $\rho=988(1-\alpha T)$ and $\ln \left[\ln \left(10^{6} \nu\right)\right]=-1.927$ $\times 10^{-3} T+0.34921$, where $\nu$ is the kinematic viscosity and where $T$ in the last expressions is the temperature in Celsius (it gives $\operatorname{Pr} \approx 880$ ). Using the thermophysical properties and the measured temperature difference, we determine the experimental Rayleigh number. The relative distance to the threshold $\epsilon=\left(\mathrm{Ra}-\mathrm{Ra}_{c}\right) / \mathrm{Ra}_{c}$ is calculated by using the critical Rayleigh number 1653 previously determined in Sec. II. Data from four different experiments were collected in which the temperature at the bottom of the fluid was fixed to 20 , 10,20 , and $40^{\circ} \mathrm{C}$ and the temperature differences were 2.41 , 5.7, 5.7, and $5.7^{\circ} \mathrm{C}$, respectively. The corresponding values of the relative distance to the threshold are $\epsilon=0.10,1.19$, 1.69 , and 2.91 , respectively. In all experiments, ten rolls parallel to the shorter sides were observed. It is well known that the wavelength increases in the nonlinear regime but in the confined problem we consider, with rather small aspect ratios and for rather small values of $\epsilon$, the number of rolls is mainly determined by the geometry $[8,18]$. The measured wave number of the eight inner rolls was 2.67 (in dimensionless units) and the coefficients of the amplitude equations were recalculated using this experimental value. The comparison between the experimental data for the different values of $\epsilon$ given above and the theoretical predictions is presented in Figs. 5 and 6, where all quantities are expressed in (dimensional) SI units. In Fig. 5, the horizontal temperature gradient is represented at midheight of the layer as a function of the 


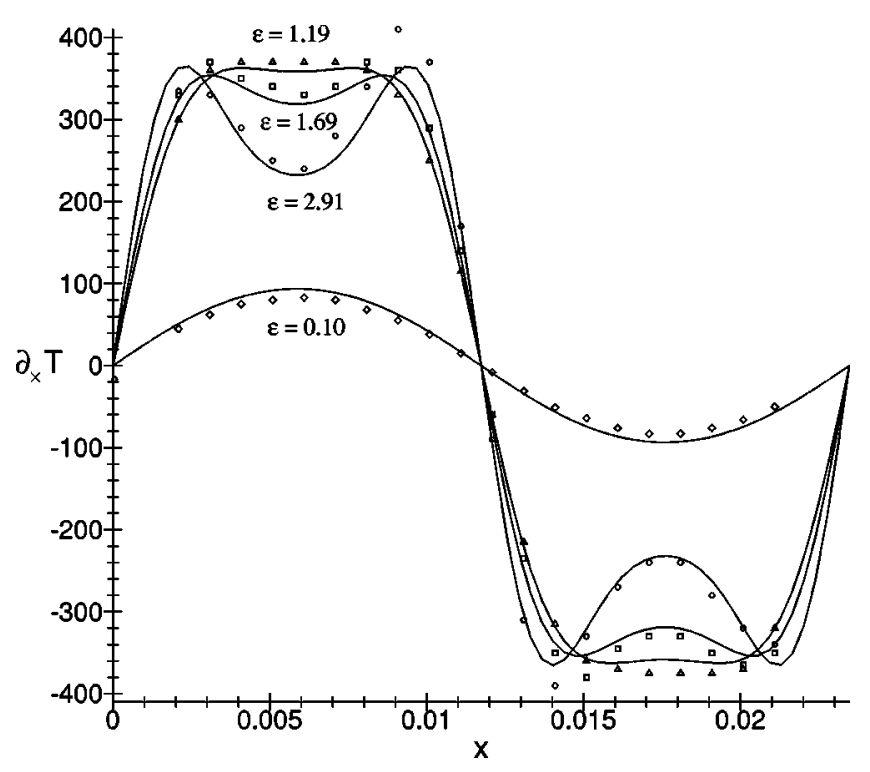

FIG. 5. Dimensional (SI units) horizontal temperature gradient $\partial_{x} T$ versus $x$ at midheight and for $\epsilon=0.1,1.19,1.69$, and 2.91. The solid curves correspond to the results of the 11 amplitude model with $k=2.67$, while the differents symbols represent the experimental data (see text for details about the experimental conditions).

horizontal coordinate. The vertical velocity is displayed in Fig. 6. The solid lines correspond to the predictions of our 11 amplitude equation model while the different symbols represent the experimental data. The agreement between our model and the experiments is very good. Besides experimental error bars, the small discrepancies may be attributed to the fact that the experimental rolls are not perfectly twodimensional while our theory does not account for threedimensional effects and for spatial variations of the thermophysical constants (computed here at the average temperature). It is interesting to note that the agreement is excellent for the velocity because the corresponding measurements were carried out in the middle of the box, where the convection is almost 2D. On the contrary, the experimental determination of the temperature gradients is based on an integral method along the rolls [12], for which the 3D aspects of the motion at both sides of the rolls have some importance.

\section{CONCLUSION}

We have shown how the amplitude method, whose validity is theoretically limited to the rather close neighborhood of the convective threshold, may be extended for larger values of $\epsilon$. An a posteriori test of the validity of the results has

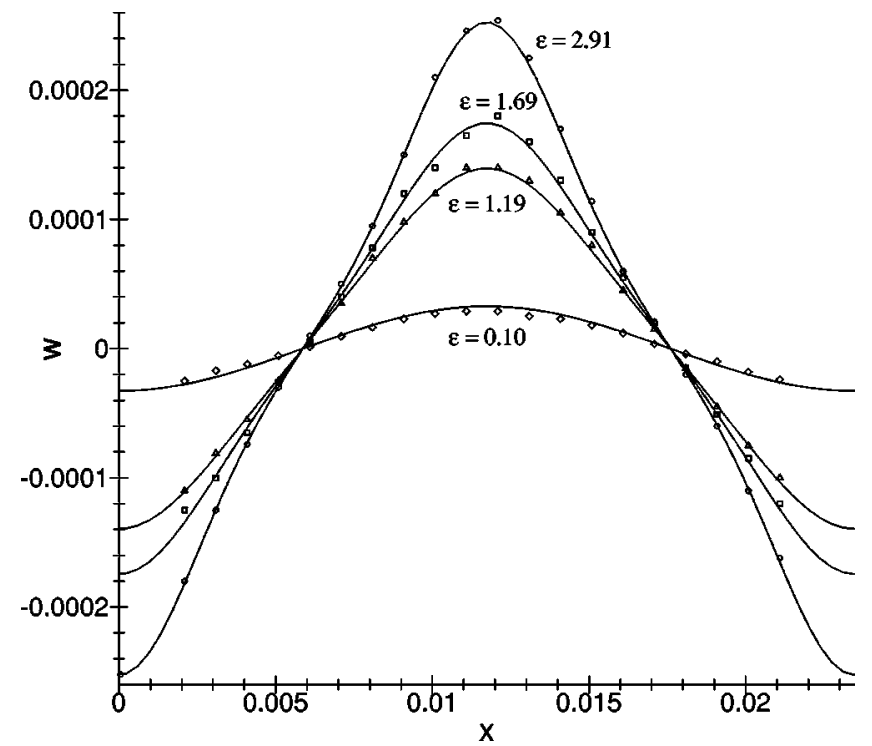

FIG. 6. Dimensional (SI units) vertical velocity $w$ versus $x$ at midheight and for $\epsilon=0.1,1.19$, and 2.91. The solid curves correspond to the results of the 11 amplitude model with $k=2.67$, while the different symbols represent the experimental data.

been provided, which consists of controlling the smallness of the amplitudes of the secondary modes. If these amplitudes are not small, the corresponding modes must be included in the basic modes and an additional amplitude equation must be considered. This procedure was used to analyze RayleighBénard convective rolls and the results were compared to direct numerical calculations as well as to experiments. In both cases, the agreement is excellent. Besides the generalized amplitude equation technique, our work provides an analysis of the appearance of spatial harmonics of the basic sine pattern in a convective flow and the actual interactions between the different modes are clearly emphasized.

\section{ACKNOWLEDGMENTS}

This paper presents results of the Belgian Program InterUniversity Pole of Attraction (IUPA 5) initiated by the Belgian State, Prime Minister's Office, Federal Office for Scientific, Technical and Cultural Affairs. Support from ESA through the CIMEX-MAP project (Contract No. 14293/00/ $\mathrm{NL} / \mathrm{SH}$ ) and from the European Union through the ICOPAC project (Contract No. HRPN-CT-2000-00136) are cordially acknowledged. It is also a pleasure to thank Professor G. Lebon for stimulating discussions as well as Professor E. Arquis and Professor J. P. Caltagirone (MASTER Laboratory, ENSCPB, Bordeaux) for providing the AQUILON code used in our numerical simulations.
[1] E.L. Koschmieder, Bénard Cells and Taylor Vortices (Cambridge University Press, Cambridge, England, 1993).

[2] P. Colinet, J. C. Legros, and M.G. Velarde, Nonlinear Dynamics of Surface Tension Driven Instabilities (Wiley-VCH, New York, 2001).
[3] P. Parmentier, V.C. Regnier, G. Lebon, and J.C. Legros, Phys. Rev. E 54, 411 (1996).

[4] V.C. Regnier, P.C. Dauby, P. Parmentier, and G. Lebon, Phys. Rev. E 55, 6860 (1997).

[5] V.C. Regnier, P.C. Dauby, and G. Lebon, Phys. Fluids 12, 2787 
(2000).

[6] P.C. Dauby, P. Colinet, and D. Johnson, Phys. Rev. E 61, 2663 (2000).

[7] J.K. Platten and J. C. Legros, Convection in Liquids (SpringerVerlag, Berlin, 1984).

[8] M. Dubois and P. Bergé, J. Fluid Mech. 85, 641 (1978).

[9] W.V.R. Malkus and G. Veronis, J. Fluid Mech. 4, 225 (1958).

[10] A. Schlüter, D. Lortz, and Busse, F., J. Fluid Mech. 23, 129 (1965).

[11] J.C. Legros and J.K. Platten, Phys. Lett. A 65A, 89 (1978).

[12] P. Cerisier, J. D. Sylvain, and P.C. Dauby, Exp. Fluids (to be published).
[13] P. Cerisier, S. Rahal, J. Cordonnier, and G. Lebon, Int. J. Heat Mass Transf. 41, 3309 (1998).

[14] C. Canuto, M.Y. Hussaini, A. Quarteroni, and T.A. Zhang, Spectral Methods in Fluid Mechanics (Springer-Verlag, Berlin 1988).

[15] P.C. Dauby and G. Lebon, J. Fluid Mech. 329, 25 (1996).

[16] P. Manneville, Dissipative Structures and Weak Turbulence (Academic Press, New York, 1990).

[17] H. Haken, Advanced Synergetics (Springer-Verlag, Berlin, 1983).

[18] K.R. Kirchartz and H. Oertel, J. Fluid Mech. 192, 249 (1988). 